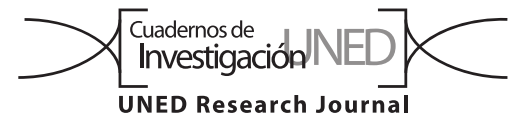

\title{
Cuantificación por absorción atómica de Cu, Fe y Zn en alcohol destilado y agua
}

\author{
Esteban Pérez López \& Diana Carlina Alvarado Rodríguez² \\ Universidad de Costa Rica, Recinto de Grecia; esteban.perezlopez@ucr.ac.cr \\ Universidad de Costa Rica, Recinto de Grecia; dianacarli_@hotmail.com
}

Recibido 03-II-2018 • Corregido 14-III-2018 • Aceptado 16-IV-2018

\begin{abstract}
Quantification by atomic absorption of $\mathrm{Cu}, \mathrm{Fe}$ and $\mathrm{Zn}$ in distilled alcohol and water. Heavy metals at the trace level have been the subject of study for several decades, however, this research focuses on copper, iron and zinc, which have been quantified in wine by the technique of atomic absorption, generally obtaining results within the permissible limits. We propose a method to analyze the indicated metals, in distilled alcoholic beverages as well as in the water used in the beverage manufacturing process. We tested acid digestion by comparing two methods, using $\mathrm{HNO} 3-\mathrm{HCl}$ and only $\mathrm{HNO}$, to reduce interferences due to organic matter in the alcohol samples; Based on the evidence and the acceptance criteria for accuracy and precision, the need for digestion with only $\mathrm{HNO} 3$ in the alcohol samples is justified. In the case of water samples, digestion is not necessary. In this way, we obtained as a result an atomic absorption methodology with the adequate performance parameters for the accurate and reliable quantification of metals in the study samples.
\end{abstract}

Key words: distilled alcoholic beverages, determination of metals, flame spectroscopy.
RESUMEN: Los metales pesados a nivel de trazas han sido objeto de estudio desde hace varias décadas, sin embargo, la presente investigación se enfoca en el cobre, hierro y zinc, los cuales se han cuantificado en vino por la técnica de absorción atómica, obteniendo generalmente resultados dentro de los límites permisibles. Proponemos un método para analizar los metales indicados, en bebidas alcohólicas destiladas así como, en el agua utilizada en el proceso de manufactura de las bebidas. Probamos la digestión ácida mediante la comparación de dos métodos, empleando $\mathrm{HNO}_{3}-\mathrm{HCl}$ y solamente $\mathrm{HNO}_{3}$, para reducir las interferencias a causa de la materia orgánica en las muestras de alcohol; con base en lo evidenciado y los criterios de aceptación para exactitud y precisión, se justifica la necesidad de digestión únicamente con $\mathrm{HNO}_{3}$ en las muestras de alcohol; $y$ en el caso de las muestras de agua no es necesaria la digestión. De esta forma, se obtuvo como resultado una metodología por absorción atómica con los parámetros de desempeño adecuados para la cuantificación veraz y confiable de los metales en las muestras de estudio.

Palabras clave: bebidas alcohólicas destiladas, determinación de metales, espectroscopía en llama.
Las bebidas alcohólicas destiladas, son aquellas que luego de ser fermentadas deben someterse a un proceso de concentración de alcohol, este proceso se denomina destilación, y a partir de dicha operación unitaria, se obtienen diversos productos como el whisky, ron, vodka y licores (Conteneo, 2015).

Al igual que la información obtenida de los estudios de metales pesados en vinos, los metales presentes en el agua potable generalmente se encuentran dentro de los límites permisibles, no obstante, el contenido de estos va a depender de la ubicación y el tipo de agua en cuestión, ya que, factores como la erosión de las rocas, depósitos minerales por las corrientes de agua, los vertimientos de las industrias y la corrosión de las tuberías transportadoras de agua, son variables a tomar en cuenta debido a que adicionan metales al cauce (Dimas, Garza \& Treviño, 2015).

Asimismo, según Simón (2008), es posible mencionar un número considerable de metales pesados como constituyentes importantes de muchas aguas, aunque su cuantificación sea a nivel de trazas. Es precisamente debido a su toxicidad, que la presencia de cualquiera de ellos en cantidades excesivas interferirá enormemente con su aplicabilidad en diversos ámbitos.

Otro aspecto importante a tomar en cuenta es la regulación vigente en el país, respecto al contenido de metales pesados presentes tanto en bebidas alcohólicas, como en el agua utilizada para consumo. El término de 
metal pesado hace referencia a cualquier elemento químico metálico que tenga una densidad relativamente alta y sea tóxico o venenoso para el organismo en concentraciones bajas. Estos elementos son componentes naturales de la corteza terrestre y no pueden ser degradados o destruidos (Gaitan, 2004).

Debido a que los metales pesados no son química ni biológicamente degradables, su peligrosidad es mucho mayor, aunado a que algunos de estos provocan efectos adversos en el cuerpo a causa de la bioacumulación. Estos se van adquiriendo y acumulando en ciertas partes ya que el organismo no logra metabolizarlos y los que están diluidos en la sangre siempre se encuentran en alguna parte causando daño en la salud, razón por la cual la ley exige un debido control de este tipo de metales en productos consumibles (Romero, 2009).

Por tal motivo, resulta de suma importancia realizar los análisis correspondientes para determinar el contenido de dichos metales, en ambos tipos de matriz. De igual forma, cabe resaltar que, las cantidades de muchos de estos metales se logran determinar a concentraciones mínimas, empleando métodos instrumentales entre los que destaca la espectroscopía de absorción atómica (Simón, 2008).

La Organización Panamericana de la Salud (1995), afirma que los metales pueden determinarse de manera satisfactoria tanto por la técnica de absorción atómica, como por colorimetría, no obstante, se indica que esta última metodología ofrece una menor precisión y sensibilidad. Además, menciona que se prefiere la medición a través del equipo de absorción atómica, ya que los resultados se obtienen con mayor rapidez y se logra evitar el empleo de técnicas tediosas para lograr la separación y eliminar las interferencias causadas por otros metales.

Asimismo, de acuerdo con estudios recientes de metales pesados mediante la técnica de absorción atómica, se ha determinado que tanto para cobre como para hierro y zinc en aguas principalmente, la metodología oficial es la técnica ya mencionada, pues la Superintendencia de Servicios Sanitarios (2007), ha creado un manual de métodos de ensayo para agua potable, en el cual se establecen las exigencias mínimas respecto a los criterios de calidad analítica que se debe alcanzar para los métodos de ensayo oficiales. Además, está basado en los estándares internacionales de la norma NCH - ISO 17025, razón por la cual, se justifica la elección de la técnica de absorción atómica en la determinación de los metales implicados, pues como lo refiere dicho manual, la técnica de absorción atómica, es el método oficial para el análisis de hierro, cobre y zinc en agua potable.
La espectrofotometría de absorción atómica, es una técnica instrumental en la cual, los átomos presentes en la llama absorben parte de la radiación, por lo tanto, la señal disminuye y ese dato es lo que mide el detector, el cual posteriormente es transformado en una concentración (Pérez, 2013). De acuerdo con la medición de la cantidad de luz absorbida, se puede hacer una determinación cuantitativa de la cantidad de analito. El uso de fuentes de luz especiales y una cuidadosa selección de las longitudes de onda permiten determinar elementos específicos (Gaitán, 2004).

Con frecuencia, la técnica de absorción atómica se utiliza en la determinación del contenido de metales pesados tanto en agua como en bebidas alcohólicas, debido a su amplia sensibilidad, ya que, como lo refiere la Organización Mundial de la Salud (2007), una de las etapas en la vigilancia de la seguridad química de los alimentos, es la obtención de datos acerca de los niveles de determinadas sustancias que pueden estar presentes en los alimentos (contaminantes, residuos, aditivos y nutrientes).

De acuerdo con los metales en estudio, cabe destacar que, el cobre es un elemento que se encuentra en la naturaleza y resulta indispensable para los seres vivos, sin embargo, se han definido una serie de patologías en las cuales se ve involucrado dicho metal, razón por la cual resulta trascendental contar con técnicas eficaces para su determinación (Soltero et al., 2009).

Por su parte, el hierro es el cuarto elemento más abundante en la corteza terrestre (5\%). La presencia del hierro en el agua provoca precipitación y coloración no deseada. Con respecto a los efectos en la salud, la sobredosis de este elemento puede provocar conjuntivitis, coriorretinitis, y retinitis si contacta con los tejidos y permanece en ellos, mientras que la inhalación crónica de concentraciones excesivas de vapores o polvos de óxido de hierro pueden provocar el desarrollo de una sideriosis (Lenntech, 2016).

De igual forma, según A.D.A.M. (2015) el zinc es un oligoelemento importante que las personas necesitan para mantenerse saludables. Entre los oligoelementos, este elemento se encuentra en segundo lugar solo después del hierro por su concentración en el organismo. No obstante, el consumo prolongado de altas dosis de zinc puede tener efectos tóxicos en la sangre (DMedicina, 2002).

Además de lo ya mencionado, un concepto que resulta necesario subrayar es el análisis de blancos, como lo mencionan Stashenko y Martínez (2011), este es reconocido como uno de los procedimientos fundamentales en la cadena analítica y de preparación de la muestra. 
Por su parte, es debido examinar las aplicaciones del ácido nítrico y sulfúrico, sobre los metales, el primero, como lo menciona Lezana y Chávarri (2008) es ampliamente utilizado cuando se quiere tener una disolución metálica ya que este disuelve a todos aquellos a quienes ataca, excepto al estaño y el antimonio que los transforma en óxidos insolubles, por esto, para ciertos usos industriales se disuelve generalmente el hierro, plomo, cobre y el zinc en ácido nítrico. Por su parte, el ácido sulfúrico es empleado en la destrucción de sustancias orgánicas (Appold, Feiler, Reinhard \& Schmidt, 2005).

Por último, con respecto a la validación de un método analítico, como lo refieren Duffau et al. (2010) este corresponde a un paso fundamental para asegurar que los resultados entregados por dicho método son confiables. Cuando se realiza la validación de un método por parte del laboratorio, lo que se busca es poder determinar con fundamento estadístico que el método es adecuado para los fines previstos.

La validación entonces corresponde al proceso mediante el cual, se logran establecer las características de desempeño y limitaciones de un método, así como la identificación de las influencias que pueden modificar esas características, lo cual debe fundamentarse mediante el suministro de evidencia objetiva (Londoño, 2013).

Dado lo anterior, y debido a la inexistencia de una metodología analítica adecuada para la cuantificación de hierro, cobre y zinc tanto en el alcohol destilado, como en el agua utilizada para la elaboración de las bebidas alcohólicas; se desarrolló el estudio evidenciado en este artículo.

De acuerdo con la norma NCR 126: 1991, dichos elementos no deben sobrepasar el límite de $10 \mathrm{mg} / \mathrm{L}$ en las bebidas, asimismo, como lo indican Dozier, McFarland y Lesikar (2005) niveles elevados de cobre pueden ocasionar un daño permanente en los riñones y causar trastornos digestivos. El exceso de hierro en los organismos puede conducir a una enfermedad fatal denominada hemosiderosis, la cual puede provocar el daño irreversible de órganos internos como el corazón, y un exceso de zinc en el cuerpo, puede interferir con la absorción de otros elementos esenciales causando anemia y problemas asociados con el estómago (Amatriain, 2000 \& Rico, 2011).

\section{METODOLOGÍA}

Para el diseño de la metodología de cuantificación de los metales pesados $\mathrm{Cu}$, Fe y $\mathrm{Zn}$, se tomó como referencia los siguientes métodos:
- AOAC Official Method 967.08. Copper in Destilled Liquors (Atomic Absorption Spectrophotometric Method)

- AOAC Official Method 970.12. Iron in Destilled Liquors (Atomic Absorption Spectrophotometric Method)

- Standard Methods for the Examination of Water and Wastewater. Metals by Flame Atomic Absorption Spectrometry (3111 B). Direct Air-Acetylene Flame Method.

- Thermo Scientific Method Iron, Copper and Zinc Determination in Wine using Flame Atomic Absorption Spectroscopy.

Los métodos de la AOAC fueron considerados para definir únicamente la concentración mínima de la curva de calibración, asimismo, del Standard Methods se tomó el procedimiento para probar el proceso de digestión ácida, y el método (no oficial) de Thermo Scientific fue empleado como la metodología base a seguir; de igual forma, se tomó en consideración las concentraciones expuestas en este para definir el rango lineal de trabajo óptimo del nuevo método a desarrollar.

Para la determinación de las condiciones óptimas de trabajo, en primera instancia, se procedió a realizar una serie de ensayos preliminares para definir tanto los parámetros de partida del instrumento, como los rangos de concentración adecuados para los dos tipos de matriz en estudio, además, se verifica si es posible la cuantificación de los metales preparados de manera conjunta (mezclados).

Con respecto a la optimización de la altura del quemador y el flujo del gas con cada elemento, se emplearon los estándares recomendados por el fabricante de Thermo Scientific (estándar de cobre de 3,5mg/L, estándar de hierro de $5 \mathrm{mg} / \mathrm{L}$ y estándar de zinc de $1 \mathrm{mg} / \mathrm{L}$ ). Posteriormente, se mide en el equipo de absorción atómica el estándar de mayor concentración de manera individual y con los otros dos elementos, para determinar la variación en la señal al mezclar los tres metales implicados.

Una vez definidos los parámetros de partida del equipo, a partir de los estándares certificados de $1000 \mathrm{mg} / \mathrm{L}$ se preparó una solución estándar de $10 \mathrm{mg} / \mathrm{L}$ con los tres metales ( $\mathrm{Cu}, \mathrm{Fe}$ y $\mathrm{Zn}$ ), la cual es diluida con ácido nítrico al $2 \% \mathrm{~m} / \mathrm{v}$ en un balón de $200 \mathrm{~mL}$. De la madre de $10 \mathrm{mg} / \mathrm{L}$ se preparan los patrones con concentraciones de 0,2 a $1,6 \mathrm{mg} / \mathrm{L}$.

Posteriormente, se realizó la medición de muestras para definir las concentraciones de analito presentes tanto en el agua como en el alcohol destilado y se procedió 
a validar los principales parámetros de desempeño del método, preparando tres curvas de calibración para evaluar la linealidad con el coeficiente de determinación arrojado en cada curva de calibración; asimismo, se evaluó el gráfico de residuales, y de manera cuantitativa mediante el test de linealidad para el coeficiente de correlación, la pendiente y el intercepto. Con respecto al criterio de precisión, se evaluó la repetibilidad, mediante seis réplicas de una muestra de agua, y seis de alcohol destilado para cada uno de los metales en estudio, las cuales han sido fortificadas con $1 \mathrm{mg} / \mathrm{L}$ de cada metal, de esta manera, se evalúa el coeficiente de variación obtenido a partir de las seis réplicas analizadas. Además, se calificó la exactitud del método mediante la preparación de seis muestras enriquecidas y no enriquecidas para determinar el \% de recuperación obtenido para cobre, hierro y zinc en las muestras de agua y el alcohol destilado. De igual forma, se toma en cuenta el sesgo obtenido, mediante el análisis de seis muestras fortificadas con $1 \mathrm{mg} / \mathrm{L}$, para determinar la veracidad de los resultados analíticos.

\section{ANÁLISIS}

Límite de detección (LOD), límite de cuantificación (LOQ) y ámbito lineal: Al comparar los coeficientes de correlación (cuadro 1) se obtuvo que el rango lineal para el zinc se ve afectado por el estándar de 0,2mg/L. Aunque la respuesta lineal a esa concentración no decrece en el caso del cobre y el hierro, el zinc también excede el máximo de aceptación para el límite de detección (LOD) y cuantificación (LOQ) (cuadro 1). Por tal motivo se modificó el intervalo de trabajo de 0,4 a 1,6mg/L (ver Fig. 1); tomando en consideración la concentración máxima permitida de los tres metales para ambos tipos de muestra, según lo refiere la norma NCR 126: 1991. Vodka y el Reglamento para la Calidad del Agua Potable vigente en Costa Rica.

Evaluación de la digestión idónea para método: El cuadro 2 evidencia la comparación del porcentaje de

CUADRO 1

Comparación de resultados y criterios de aceptación para definir el intervalo lineal óptimo de trabajo, así como el LOD y LOQ para cada metal

\begin{tabular}{llcccccc} 
Analito & \multicolumn{1}{c}{ Detalle } & \multicolumn{3}{c}{ Curva de 0,2 a 1,6mg/L } & \multicolumn{3}{c}{ Curva de 0,4 a 1,6mg/L } \\
Cobre & Especificación & $r^{2}(\geq 0,995)$ & $\operatorname{LOD}(\leq 0,200)$ & $\operatorname{LOQ}(\leq 0,200)$ & $r^{2}(\geq 0,995)$ & $\operatorname{LOD}(\leq 0,200)$ & $\operatorname{LOQ}(\leq 0,200)$ \\
& Resultado & 0,999 & 0,070 & 0,200 & 0,999 & 0,040 & 0,130 \\
Hierro & Especificación & $r^{2}(\geq 0,995)$ & $\operatorname{LOD}(\leq 0,030)$ & $\operatorname{LOQ}(\leq 0,060)$ & $r^{2}(\geq 0,995)$ & $\operatorname{LOD}(\leq 0,030)$ & $\operatorname{LOQ}(\leq 0,060)$ \\
& Resultado & 1,000 & 0,020 & 0,050 & 1,000 & 0,020 & 0,050 \\
Zinc & Especificación & $r^{2}(\geq 0,995)$ & $\operatorname{LOD}(\leq 0,200)$ & $\operatorname{LOQ}(\leq 0,200)$ & $r^{2}(\geq 0,995)$ & $\operatorname{LOD}(\leq 0,200)$ & $\operatorname{LOQ}(\leq 0,200)$ \\
& Resultado & $0,990^{*}$ & $0,230^{*}$ & $0,710^{*}$ & 0,998 & 0,07 & 0,200 \\
\hline
\end{tabular}

*No cumple con criterio de aceptación establecido.

CUADRO 2

Porcentaje de recuperación de $\mathrm{Cu}$, Fe y $\mathrm{Zn}$ en muestras de agua y alcohol destilado, utilizando $\mathrm{HNO}_{3}$ y $\mathrm{HNO}_{3}-\mathrm{HCl}$ en la digestión

\begin{tabular}{|c|c|c|c|c|}
\hline \multicolumn{5}{|c|}{ Muestras digeridas $\mathrm{HNO}_{3}-\mathrm{HCl}$} \\
\hline \multicolumn{3}{|c|}{ Alcohol Destilado } & \multicolumn{2}{|c|}{ Agua Potable } \\
\hline Analito & $\% \mathbf{R}$ & RDS & $\% \mathbf{R}$ & RDS \\
\hline Cobre & 87 & 9,25 & 91 & 6,91 \\
\hline Hierro & 52 & 74,36 & 88 & 1,91 \\
\hline Zinc & 94 & 17,17 & 114 & 14,56 \\
\hline \multicolumn{5}{|c|}{ Muestras digeridas $\mathrm{HNO}_{3}$} \\
\hline \multicolumn{3}{|c|}{ Alcohol Destilado } & \multicolumn{2}{|c|}{ Agua Potable } \\
\hline Analito & $\% R$ & RDS & $\% \mathbf{R}$ & RDS \\
\hline Cobre & 99 & 1,02 & 96 & 3,18 \\
\hline Hierro & 101 & 2,35 & 93 & 13,19 \\
\hline Zinc & 104 & 2,35 & 105 & 0,70 \\
\hline
\end{tabular}

\%R: Porcentaje de Recuperación; RSD: Desviación Estándar Relativa. 


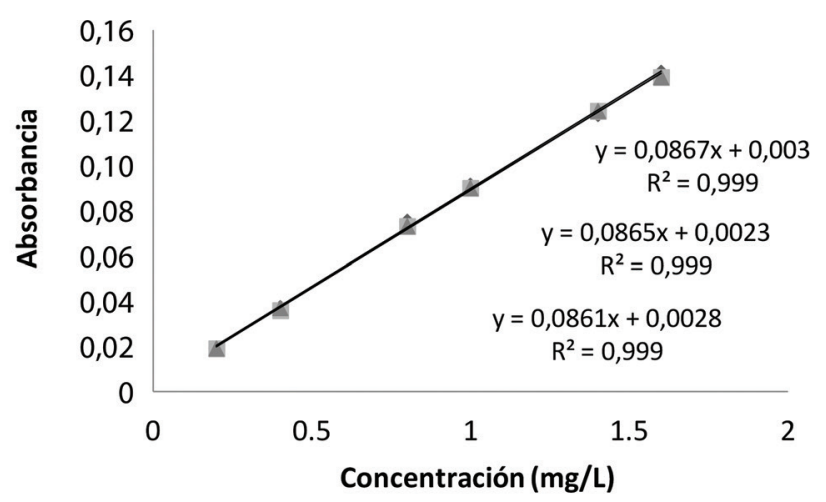

Fig. 1. Superposición de las curvas de calibración para el cobre.

recuperación tanto en las muestras digeridas, como en las no digeridas para el alcohol destilado y el agua potable, empleando $\mathrm{HNO}_{3}-\mathrm{HCl}$ y solamente $\mathrm{HNO}_{3}$ durante el proceso de digestión. Obteniendo como resultado el incumplimiento del criterio de aceptación para exactitud según la recuperación de metal añadido para hierro y cobre, en las muestras de alcohol destilado digerido con $\mathrm{HNO}_{3}-\mathrm{HCl}$, pues como lo indica el Codex Comittee on Methods of Analysis and Sampling (2009) este debe ser de $90-110 \%$.

En el caso del hierro y el zinc, tampoco se cumple con el criterio de aceptación para precisión, según lo establecido por Clesceri, Greenberg y Eaton (1999), debido a que la desviación estándar relativa supera el 10\%. Además, se evidencia que en el caso del hierro y el zinc para las muestras de agua digeridas con $\mathrm{HNO}_{3}-\mathrm{HCl}$, se incumple en exactitud, y según los datos obtenidos para este último metal, también en precisión. No obstante, se observa una mejoría en las muestras de alcohol al realizar el proceso de digestión empleando únicamente $\mathrm{HNO}_{3^{\prime}}$ ya que los tres metales implicados se encuentran dentro del criterio establecido tanto para exactitud, como para precisión. Con respecto a las muestras de agua digeridas solamente con $\mathrm{HNO}_{3^{\prime}}$ de igual manera presentan un comportamiento más estable, sin embargo, en el caso del hierro no se cumple con el criterio de aceptación para precisión.

El Laboratorio Nacional de Referencia para Metales Pesados en Alimentos y Piensos (2012), para las bebidas alcohólicas de alta graduación con bajo contenido de azúcares $(<50 \mathrm{~g} / \mathrm{L})$, resulta necesario evaporar el alcohol mediante calentamiento hasta casi sequedad, para eliminar interferencias de este tipo. Asimismo, como lo refiere Constanza y Restrepo (2011), la materia orgánica presente en muestras de bebidas alcohólicas se elimina fácilmente por digestión.

Con respecto al medio ácido a utilizar en el proceso de digestión de las muestras de alcohol, resultó mejor el ácido nítrico, pues como lo respaldan Clesceri et al (1999), se utiliza el procedimiento de digestión menos riguroso requerido, para proporcionar una recuperación aceptable y consistente, compatible con el método analítico y el metal que se analiza. La digestión con $\mathrm{HNO}_{3}$ arrojó una mejor recuperación para el cobre, el hierro y el zinc, además de menor variabilidad en los resultados.

Aunque algunas muestras requieren la adición de otros ácidos como perclórico o sulfúrico para completar la digestión, estos también pueden generar interferencias durante el análisis de algunos metales. Lo anterior se debe a que la matriz se transforma en una de mayor complejidad, lo cual podría ser una causa de los confusos resultados generados en el caso de la digestión al emplear $\mathrm{HNO}_{3}-\mathrm{HCl}$, de igual forma, dicha combinación de ácidos se recomienda para analizar metales como: plata, antimonio, rutenio y estaño, es decir, ninguno de los metales implicados. (Clesceri et al., 1999).

También, se probó la determinación de los metales en las muestras de alcohol y agua sin digerir (cuadro 3), $y$ aunque en el alcohol se cumplen con los criterios de precisión, se demuestran porcentajes de recuperación menores al 80\%; contrario a lo que sucede en el caso del agua, que los resultados fueron más precisos, y la recuperación dentro de lo deseable (90-110\%). Clesceri et al (1999), afirman que muestras incoloras y transparentes

\section{CUADRO 3}

Porcentaje de recuperación en muestras de agua y alcohol destilado sin el proceso de digestión

\begin{tabular}{|c|c|c|c|c|}
\hline \multicolumn{5}{|c|}{ Muestras no digeridas } \\
\hline \multicolumn{3}{|c|}{ Alcohol Destilado } & \multicolumn{2}{|c|}{ Agua Potable } \\
\hline Analito & $\% R$ & RDS & $\% R$ & RDS \\
\hline Cobre & 73 & 1,45 & 104 & 0,79 \\
\hline Hierro & 78 & 1,83 & 93 & 1,72 \\
\hline Zinc & 76 & 5,05 & 103 & 1,22 \\
\hline
\end{tabular}

\%R: Porcentaje de Recuperación; RSD: Desviación Estándar Relativa. 
(agua potable de primer nivel) con una turbidez $<1 \mathrm{NTU}$, sin olor y de una sola fase, pueden ser analizadas directamente mediante espectroscopía de absorción atómica para metales totales sin digestión. No obstante, dichos autores enfatizan en la necesidad de realizar una verificación adicional donde se contrasten muestras digeridas y no digeridas para asegurar resultados comparables.

Linealidad del método analítico: Con respecto a la validación del parámetro de linealidad del método, en el cuadro 4 se determinó que el análisis de cobre cumple con el test de linealidad para el coeficiente de correlación, debido a que en la prueba t-student aplicada el $t$ exp > t tab, lo cual indica que se rechaza la hipótesis nula Ho, es decir, la correlación lineal es estadísticamente significativa con la probabilidad calculada. Asimismo, en el caso de la pendiente se observa que el $t \exp >t t a b$, la pendiente difiere significativamente de cero, al igual que en el test del intercepto. En cuanto a este último criterio, si bien es cierto, lo ideal es que el intercepto difiera del cero lo menos posible, es decisión del laboratorio aceptar o rechazar la linealidad al verse afectada por este parámetro, ya que el mismo no es determinante en el análisis, en caso de que los demás parámetros hayan sido aceptados.
En la Fig. 2, se estima que existe aleatoriedad en el análisis de los residuos, como lo refiere Magnusson y Örnemark (2014), la distribución aleatoria de residuales en torno a cero confirma la linealidad, mientras que las tendencias sistemáticas indican la no linealidad o un cambio de varianza con el nivel. Por último, cabe destacar que el coeficiente de variación de los factores de respuesta es inferior a 10\%, tal como lo indica el Codex Comittee on Methods of Analysis and Sampling (2009), por lo tanto, en términos generales, es posible afirmar que en el análisis de cobre se cumple con el criterio de linealidad.

Los resultados descritos en el cuadro 4, demuestran que el análisis de hierro cumple con el test de linealidad para el coeficiente de correlación, debido a que el t exp > t tab, lo cual indica que se rechaza la hipótesis nula Ho. La correlación lineal es estadísticamente significativa con la probabilidad calculada. Asimismo, en el caso de la pendiente se observa que el $t \exp >t t a b$, la pendiente difiere significativamente de cero, contrario a lo que sucede en el test del intercepto, pues texp $<t t a b$, es decir, el intercepto no difiere significativamente de cero, por tal motivo, también cumple con dicho criterio.

En la Fig. 3, se observa que existe aleatoriedad en el análisis de los residuos, lo cual confirma que existe linealidad. Además, cabe destacar que el coeficiente de

\section{CUADRO 4}

Resultados del estudio de linealidad para los tres metales

\begin{tabular}{lccc}
\multicolumn{1}{c}{ Especificación } & Cobre & Hierro & Zinc \\
Coeficiente de correlación & $0,999>0,995$ & $1,000>0,995$ & $0,999>0,995$ \\
Test de linealidad para la Pendiente & $\mathrm{t}_{\exp } 123,98>\mathrm{t}_{\text {tab }} 2,16$ & $\mathrm{t}_{\exp } 173,04>\mathrm{t}_{\text {tab }} 2,16$ & $\mathrm{t}_{\exp } 72,82>\mathrm{t}_{\text {tab }} 2,16$ \\
Test de linealidad para el Intercepto & $\mathrm{t}_{\exp } 3,34^{*}<\mathrm{t}_{\text {tab }} 2,16$ & $\mathrm{t}_{\text {exp }} 0,96<\mathrm{t}_{\text {tab }} 2,16$ & $\mathrm{t}_{\text {exp }} 5,97^{*}<\mathrm{t}_{\text {tab }} 2,16$ \\
Aleatoriedad en el análisis de los residuos & Existe aleatorieda & Existe aleatoriedad & Existe aleatoriedad \\
Coeficiente de variación & $3 \%<10 \%$ & $2 \%<10 \%$ & $7 \%<10 \%$ \\
\hline
\end{tabular}

*No cumple con criterio de aceptación establecido.

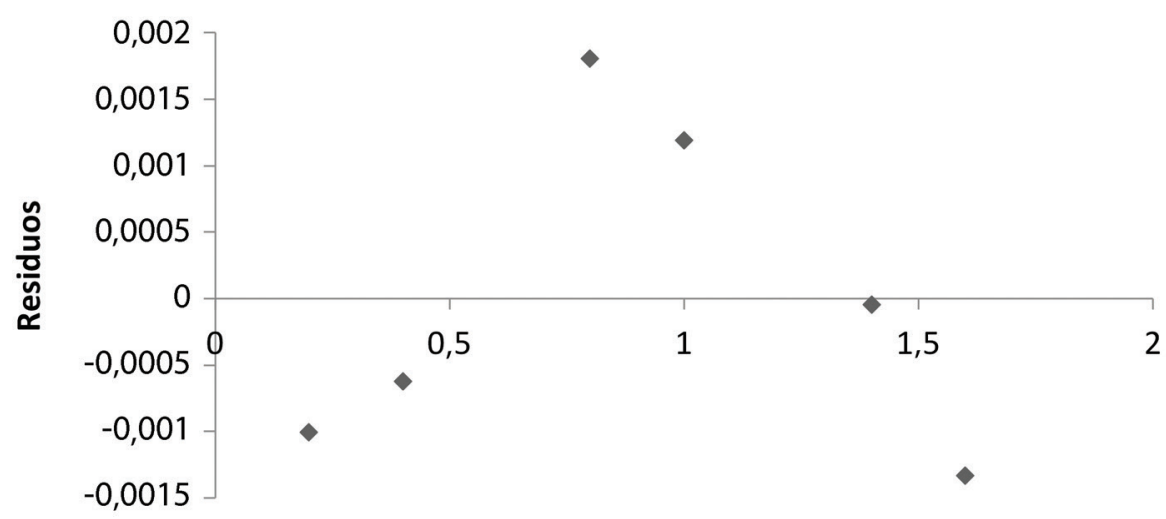

Fig. 2. Análisis de Residuales para la curva de calibración de cobre. 


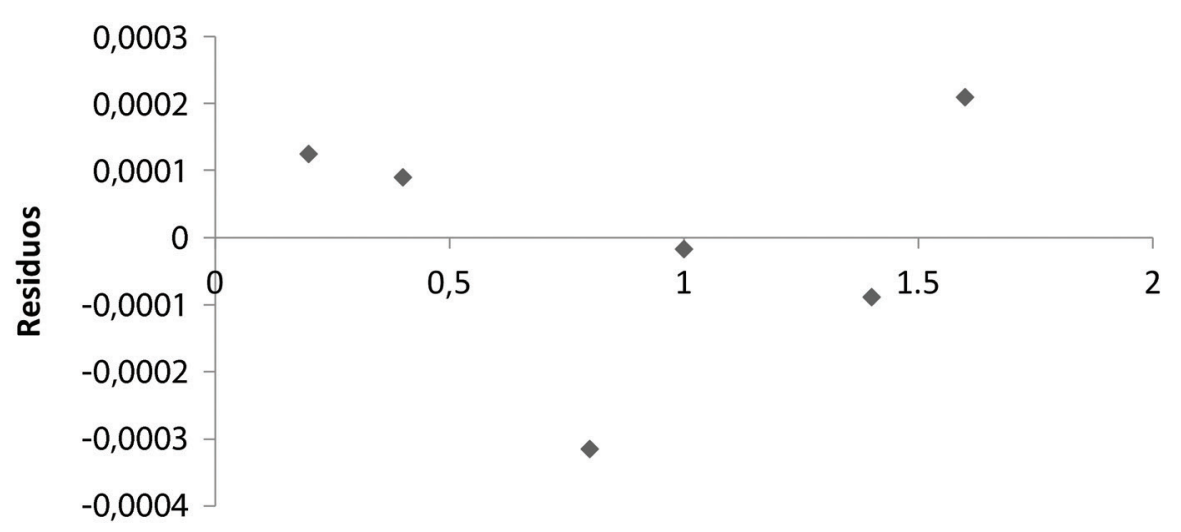

Fig. 3. Análisis de Residuales para la curva de calibración de hierro.

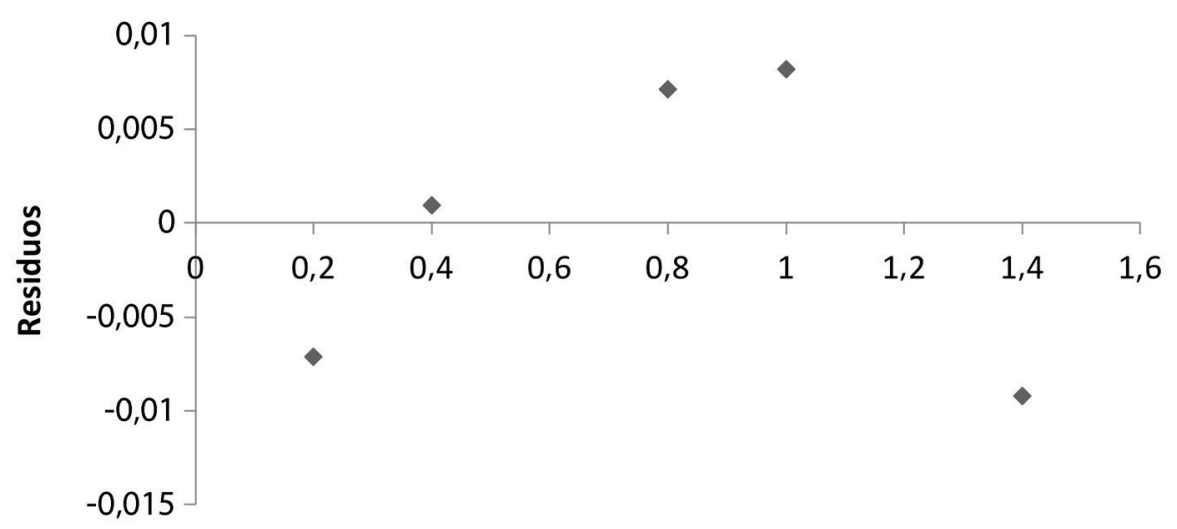

Fig. 4. Análisis de residuales para la curva de calibración de zinc.

variación de los factores de respuesta es inferior a $10 \%$, por lo tanto, es posible afirmar que en el análisis de Hierro se cumple con el criterio de Linealidad.

Igualmente, los expuesto en el cuadro 4 evidencia que el análisis de zinc cumple con el test de linealidad para el coeficiente de correlación, debido a que el $t$ exp $>t$ tab, lo cual indica que se rechaza la hipótesis nula. En el caso de la pendiente se observa que el $t \exp >t t a b$, la pendiente difiere significativamente de cero, al igual que en el test del intercepto. Sin embargo, como se mencionó en el caso del cobre, debido a que los demás parámetros cumplen con los criterios de aceptación, es decisión del laboratorio si se acepta o rechaza la linealidad al verse afectada por este parámetro, ya que el mismo no es determinante en el análisis. En la Fig. 4 se demuestra que existe aleatoriedad en el análisis de los residuos, por lo que se justifica la linealidad del modelo. Con respecto al coeficiente de variación de los factores de respuesta, este es inferior a $10 \%$. Por tal razón, el análisis de zinc cumple con el criterio de linealidad.
Precisión y exactitud del método: Para determinar la precisión del método, se evaluó la repetibilidad de las mediciones en el análisis de cobre, hierro y zinc (cuadros 5,6 y 7), a partir de seis muestras fortificadas con $1 \mathrm{mg} / \mathrm{L}$ de cada metal. El coeficiente de variación para cada análisis de agua y alcohol es inferior al coeficiente de variación porcentual de Horwitz, ya que para la concentración de $1 \mathrm{mg} / \mathrm{L}$, dicho coeficiente no debe superar el $8 \%$, lo cual indica que se cumple con el criterio establecido para repetibilidad a esa concentración.

En cuanto a la determinación del sesgo (exactitud) en las muestras de agua para el cobre (cuadro 5), se arroja un $t$ calc $>t$ crit, lo que indica que en el caso de estas muestras si existen diferencias significativas. Pero, utilizando el criterio relativo del valor obtenido con respecto al valor real, este es menor al 10\%, por lo cual, siempre se considera aceptable el sesgo para el método, sin que esto genere alteración para los fines previstos de la metodología.

Por su parte, tanto para el hierro (Cuadro 6), como para el zinc (Cuadro 7), en ambas matrices estudiadas el $t$ calc 


\section{CUADRO 5}

Resultados de repetibilidad y exactitud del método para cobre

\begin{tabular}{|c|c|c|c|c|c|}
\hline \multicolumn{2}{|c|}{ Muestras de Agua } & \multicolumn{4}{|c|}{ Cobre } \\
\hline \multirow{2}{*}{ Repetición } & \multirow{2}{*}{$\mathrm{mg} / \mathrm{L}$} & \multirow{2}{*}{$\begin{array}{c}C V \\
\leq 8,00 \%\end{array}$} & \multirow{2}{*}{$\begin{array}{c}\text { Sesgo } \\
\text { tcalc }<\text { tcrit }\end{array}$} & \multicolumn{2}{|c|}{ Recuperación } \\
\hline & & & & $(90-110) \%$ & RSD $<10 \%$ \\
\hline 1 & 1,07 & \multirow{6}{*}{$0,76 \%$} & \multirow{6}{*}{$\begin{array}{c}0,01 \\
\text { tcalc }>\text { tcrit }\end{array}$} & \multirow{6}{*}{$104 \%$} & \multirow{6}{*}{$0,79 \%$} \\
\hline 2 & 1,07 & & & & \\
\hline 3 & 1,06 & & & & \\
\hline 4 & 1,06 & & & & \\
\hline 5 & 1,06 & & & & \\
\hline 6 & 1,08 & & & & \\
\hline \multicolumn{2}{|c|}{ Muestras de Alcohol } & \multicolumn{4}{|c|}{ Cobre } \\
\hline \multirow{2}{*}{ Repetición } & \multirow{2}{*}{$\mathrm{mg} / \mathrm{L}$} & \multirow{2}{*}{$\begin{array}{c}\text { CV\% } \\
\leq 8,00 \%\end{array}$} & \multirow{2}{*}{$\begin{array}{c}\text { Sesgo } \\
\text { tcalc }>\text { tcrit }\end{array}$} & \multicolumn{2}{|c|}{ Recuperación } \\
\hline & & & & $(90-110) \%$ & RSD $<10 \%$ \\
\hline 1 & 0,99 & \multirow{6}{*}{$0,89 \%$} & \multirow{6}{*}{$\begin{array}{c}0,01 \\
\text { tcalc }>\text { tcrit }\end{array}$} & \multirow{6}{*}{$99 \%$} & \multirow{6}{*}{$1,02 \%$} \\
\hline 2 & 0,99 & & & & \\
\hline 3 & 0,99 & & & & \\
\hline 4 & 1,00 & & & & \\
\hline 5 & 1,00 & & & & \\
\hline 6 & 1,01 & & & & \\
\hline
\end{tabular}

\section{CUADRO 6}

Resultados de repetibilidad y exactitud del método para hierro

\begin{tabular}{|c|c|c|c|c|c|}
\hline \multicolumn{2}{|c|}{ Muestras de Agua } & \multicolumn{4}{|c|}{ Hierro } \\
\hline \multirow{2}{*}{ Repetición } & \multirow{2}{*}{$\mathrm{mg} / \mathrm{L}$} & \multirow{2}{*}{$\mathrm{CV} \leq 8,00 \%$} & \multirow{2}{*}{ Sesgo tcalc $<$ tcrit } & \multicolumn{2}{|c|}{ Recuperación } \\
\hline & & & & $(90-110) \%$ & $\mathrm{RSD}<10 \%$ \\
\hline 1 & 0,92 & \multirow{6}{*}{$1,72 \%$} & \multirow{6}{*}{0,02 tcalc $<$ tcrit } & \multirow{6}{*}{$93 \%$} & \multirow{6}{*}{$1,72 \%$} \\
\hline 2 & 0,92 & & & & \\
\hline 3 & 0,92 & & & & \\
\hline 4 & 0,93 & & & & \\
\hline 5 & 0,94 & & & & \\
\hline 6 & 0,96 & & & & \\
\hline \multicolumn{2}{|c|}{ Muestras de Alcohol } & \multicolumn{4}{|c|}{ Hierro } \\
\hline \multirow{2}{*}{ Repetición } & \multirow{2}{*}{$\mathrm{mg} / \mathrm{L}$} & \multirow{2}{*}{ CV\% $\leq 8,00 \%$} & \multirow{2}{*}{ Sesgo tcalc $<$ tcrit } & \multicolumn{2}{|c|}{ Recuperación } \\
\hline & & & & $(90-110) \%$ & RSD $<10 \%$ \\
\hline 1 & 1,02 & \multirow{6}{*}{$2,36 \%$} & \multirow{6}{*}{0,02 tcalc $<$ tcrit } & \multirow{6}{*}{$101 \%$} & \multirow{6}{*}{$2,35 \%$} \\
\hline 2 & 1,04 & & & & \\
\hline 3 & 1,01 & & & & \\
\hline 4 & 0,97 & & & & \\
\hline 5 & 1,03 & & & & \\
\hline 6 & 1,01 & & & & \\
\hline
\end{tabular}




\section{CUADRO 7}

Resultados de los parámetros repetibilidad y exactitud del método para zinc

\begin{tabular}{|c|c|c|c|c|c|}
\hline \multicolumn{2}{|c|}{ Muestras de Agua } & \multicolumn{4}{|c|}{ Zinc } \\
\hline \multirow{2}{*}{ Repetición } & \multirow{2}{*}{$\mathrm{mg} / \mathrm{L}$} & \multirow{2}{*}{$\mathrm{CV} \leq 8,00 \%$} & \multirow{2}{*}{ Sesgo tcalc $<$ tcrit } & \multicolumn{2}{|c|}{ Recuperación } \\
\hline & & & & $(90-110) \%$ & RSD $<10 \%$ \\
\hline 1 & 1,03 & \multirow{6}{*}{$1,22 \%$} & \multirow{6}{*}{$\begin{array}{c}0,01 \\
\text { tcalc }<\text { tcrit }\end{array}$} & \multirow{6}{*}{$103 \%$} & \multirow{6}{*}{$1,22 \%$} \\
\hline 2 & 1,04 & & & & \\
\hline 3 & 1,04 & & & & \\
\hline 4 & 1,04 & & & & \\
\hline 5 & 1,02 & & & & \\
\hline 6 & 1,01 & & & & \\
\hline \multicolumn{2}{|c|}{ Muestras de Alcohol } & \multicolumn{4}{|c|}{ Zinc } \\
\hline \multirow{2}{*}{ Repetición } & \multirow{2}{*}{$\mathrm{mg} / \mathrm{L}$} & \multirow{2}{*}{$C V \% \leq 8,00 \%$} & \multirow{2}{*}{ Sesgo tcalc $<$ tcrit } & \multicolumn{2}{|c|}{ Recuperación } \\
\hline & & & & $(90-110) \%$ & RSD $<10 \%$ \\
\hline 1 & 1,02 & \multirow{6}{*}{$2,37 \%$} & \multirow{6}{*}{$\begin{array}{c}0,02 \\
\text { tcalc }<\text { tcrit }\end{array}$} & \multirow{6}{*}{$104 \%$} & \multirow{6}{*}{$2,36 \%$} \\
\hline 2 & 1,04 & & & & \\
\hline 3 & 1,01 & & & & \\
\hline 4 & 1,06 & & & & \\
\hline 5 & 1,05 & & & & \\
\hline 6 & 1,08 & & & & \\
\hline
\end{tabular}

$<t$ crit, lo cual significa que el sesgo obtenido y su veracidad es aceptable.

En la evaluación de la capacidad de recuperación del método se evidencia el cumplimiento de dicho criterio para ambos tipos de muestras en los tres metales, lo que genera confianza en los resultados de exactitud. El porcentaje de recuperación de cada metal se encuentra entre el $90-110 \%$, por lo que, cumplen con el criterio de aceptación establecido por el Codex Comittee on Methods of Analysis and Sampling (2009).

\section{AGRADECIMIENTOS}

Agradecemos al personal del Laboratorio de Control de Calidad de la Fábrica Nacional de Licores en Costa Rica, por la apertura para realizar trabajos como este, y por todo el apoyo brindado durante el proceso de indagación.

\section{REFERENCIAS}

A.D.A.M. (2015). MedlinePlus Información de Salud. Recuperado de: $\quad$ https://medlineplus.gov/spanish/ency/article/002416.htm

Amatriain, M. (2000). Efectos del exceso de hierro sobre la salud. Medicina Naturista, 1(2), 92-95.
Appold, H., Feiler, K., Reinhard, A., y Schmidt, P. (2005). Tecnología de los Metales. Barcelona: Reverte. Recuperado de : http://goo.gl/9dpwNL

Clesceri, L., Greenberg, A., y Eaton, A. (1999). Standard Methods for the Examination of Water and Wastewater. 20 th edition. United States of America: American Public Health Association.

Codex Comittee on Methods of Analysis and Sampling. (2009). Guidelines on Analytical Terminology (cac/gl72-2009).

Constanza, D. y Restrepo, M. (2011). Validación de las metodologías en el Laboratorio de Aguas y Alimentos de la Universidad Tecnológica de Pereira para la Determinación de Hierro y Cobre en Muestras de Ron provenientes de la Industria Licorera de Caldas por la Técnica de Absorción Atómica en llama.

Conteneo S. L. (2015). Infodrinks. Ercilla: Conteneo Networks S.L. Recuperado de: http://es.infodrinks.com/

Dimas, M., Garza, M., Treviño, D. (2015). Índice de la calidad del agua y metales pesados del cauce aguas blancas del municipio de Acapulco Guerrero, México. Revista Mexicana de Ciencias Agrícolas, 1(1), 113-118. Recuperado de: http://www.redalyc.org/articulo.oa?id=263139243016

DMedicina. (2002). DMedicina.com Salud y Bienestar. Recuperado de: http://www.dmedicina.com/vida-sana/ deporte/2002/11/27/suplementos-zinc-efecto-toxico-4976.html

Dozier, M. McFarland, M. y Lesikar, B. (2005). Problemas del agua potable: El cobre. Texas: Comunicaciones Agrícolas, El Sistema Universitario Texas A\&M. Recuperado de: goo. $\mathrm{gl} / 8 \mathrm{RnFBd}$. 
Duffau, B., Rojas, F., Guerrero, I., Roa, L., Rodríguez, L., Soto, M., Aguilera, M., y Sandoval, S. (2010). Validación de métodos y determinación de la incertidumbre de la medición: "Aspectos generales sobre la validación de métodos". Santiago.

Fernández, V.; Berradre, M.; Sulbarán, B.; Rodríguez, G. y Peña, J. (2009). Caracterización química y contenido mineral en vinos comerciales venezolanos. Rev. Fac. Agron. 26(3). 382-397. Recuperado de: http:// www.scielo.org.ve/scielo.php?script=sci_arttext\&pi$\mathrm{d}=$ S0378-78182009000300005

Gaitan, M. (2004). Determinación de Metales Pesados totales con digestión ácida y solubles lectura directa por Espectrofotometría de Absorción Atómica. Instituto de Hidrología, Meteorología y Estudios Ambientales. República de Colombia. Recuperado de: file:///E:/ Proyecto\%20Industrial/Metales\%20en\%20agua\%20 por\%20Absorción\%20Atómica.\%20INTERFERENCIAS. pdf

Laboratorio Nacional de Referencia para Metales Pesados en Alimentos y Piensos. (2012). Análisis de elementos para categorías de ensayo NT-18. Mediante las técnicas de Espectrometría Atómica o Espectrometría de Masas. Recuperado de: file:///E:/Proyecto\%20Industrial/explicación\%20de\%20acidos\%20sólo\%20evaporar\%20 el\%20alcohol.pdf

Lenntech B.V. (2016). Water Treatment. Recuperado de: https:// www.lenntech.com/water-treatment.htm

Lezana, G. y Chávarri, J. (2008). Elementos de química: con sus principales aplicaciones a la medicina, a las artes y a la industria. The Complutense University of Madrid. Recuperado de: http://goo.gl/NJef4d

Londoño, D. (2013). Validación del método de determinación de calcio y magnesio por espectroscopia de absorción atómica de llama para el laboratorio de análisis de aguas y alimentos de la Universidad Tecnológica de Pereira. Recuperado de: file:///E:/Proyecto\%20Industrial/ Método\%20Validación\%20Ca\%20y\%20Mg.pdf

Magnusson, B. y Örnemark, U. (2014), Eurachem Guide: The Fitness for Purpose of Analytical Methods - A Laboratory Guide to Method Validation and Related Topics. 2 edición. Recuperado de: www.eurachem.org
Organización Mundial de la Salud. (2007). Manual sobre las cinco claves para la inocuidad de los alimentos. Francia: Ediciones de la OMS. Recuperado de: http:// www.who.int/foodsafety/publications/consumer/ manual_keys_es.pdf

Organización Panamericana de la Salud. (1995). Análisis de metales en agua potable y residual por espectrofotometría de Absorción Atómica. Lima: Centro Panamericano de Ingeniería Sanitaria y Ciencias del Ambiente. Recuperado de: http://www.bvsde.paho.org/bvsacg/ fulltext/analisis.pdf

Pérez, E. (2013). Manual de Laboratorio de Técnicas Instrumentales de Análisis. Tacares de Grecia: Universidad de Costa Rica.

Rico, J. (2011). La actividad física y el zinc: una revisión. Archivos de Medicina del Deporte, 28(141), 36-44.

Romero, K. (2009). Contaminación por metales pesados. Revista Científica Ciencia Médica, 12(1), 45-46. Recuperado de: http://www.scielo.org.bo/pdf/rccm/v12n1/v12n1_a13. pdf

Simón, E. (2008). Los metales pesados en las aguas residuales. Madrid. Recuperado de: http://www.madrimasd.org/ blogs/remtavares/2008/02/02/83698

Soltero, D., Palafox, S., Herrera, R., Dávila, A., Cuéllar, J., y González, M. (2009). Determinación de niveles de Cobre en una población estudiantil de la Facultad de Ciencias Químicas por espectrofotometría de absorción atómica en flama. Synthesis. 1-5. http://goo.gl/MCWQUS

Stashenko, E. y Martínez, J. (2011). Preparación de la muestra: un paso crucial para el análisis por GC-MS. Scientia Chromatographica, 3 (1). 25-49, Recuperado de: http:// www.scientiachromatographica.com/files/v3n1/ v3n1a3.pdf

Superintendencia de Servicios Sanitarios. (2007). Manual de métodos de ensayo para agua potable. Recuperado de: http://www.siss.gob.cl/586/w3-article-9648.html 